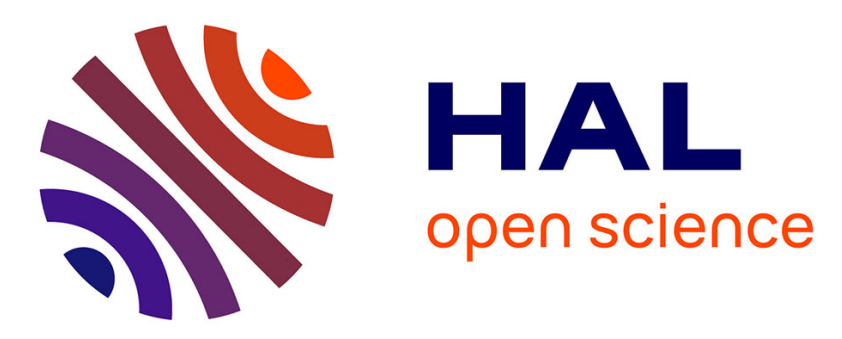

\title{
Modeling and experimental characterization of an active MEMSbased force sensor
}

\author{
Jonathan Cailliez, Mokrane Boudaoud, Abdenbi Mohand Ousaid, Antoine \\ Duflos, Sinan Haliyo, Stéphane Regnier
}

\section{To cite this version:}

Jonathan Cailliez, Mokrane Boudaoud, Abdenbi Mohand Ousaid, Antoine Duflos, Sinan Haliyo, et al.. Modeling and experimental characterization of an active MEMSbased force sensor. Journal of Micro-Bio Robotics, 2019, 15 (1), pp.53 - 64. hal-02867735

\section{HAL Id: hal-02867735 \\ https://hal.science/hal-02867735}

Submitted on 15 Jun 2020

HAL is a multi-disciplinary open access archive for the deposit and dissemination of scientific research documents, whether they are published or not. The documents may come from teaching and research institutions in France or abroad, or from public or private research centers.
L'archive ouverte pluridisciplinaire HAL, est destinée au dépôt et à la diffusion de documents scientifiques de niveau recherche, publiés ou non, émanant des établissements d'enseignement et de recherche français ou étrangers, des laboratoires publics ou privés. 


\title{
Modeling and experimental characterization of an active MEMS based force sensor
}

\author{
Jonathan Cailliez ${ }^{1} \cdot$ Mokrane Boudaoud $^{1} \cdot$ Abdenbi Mohand-Ousaid $^{2} \cdot$ Antoine Weill-Duflos $^{3} \cdot$ Sinan Haliyo $^{1}$. \\ Stéphane Régnier ${ }^{1}$
}

Received: 19 October 2018 / Revised: 9 January 2019 / Accepted: 11 March 2019

(C) Springer-Verlag GmbH Germany, part of Springer Nature 2019

\begin{abstract}
Active force sensors are based on the principle of force balancing using a feedback control. They allow, unlike passive sensors, the static characterization of forces without interference of the sensor mechanical properties on the estimated stiffness of the object to be studied. This capability is fundamental when dealing with the mechanical characterization of samples having a wide range of stiffness. This paper deals with the modeling and the experimental characterization of a new active MEMS based force sensor. This sensor includes folded-flexure type suspensions and a differential comb drive actuation allowing a linear force/voltage relationship. A control oriented electromechanical model is proposed and validated experimentally in static and dynamic operating modes using a stroboscopic measurement system. This work is a first step towards new MEMS active force sensor with high resonant frequency $(>2 \mathrm{kHz})$ and high linear measurement force range $(50 \mu N)$. The advantage of this structure is to be able to change the sensor operating point without changing the sensor dynamics. Thus simplifying the control law. Modifying the operating point allows performing an accurate self positioning of the probe in close proximity to the surface to be studied.
\end{abstract}

\section{Keywords}

\section{Introduction}

Small and embeddable force sensing tools are essential in micro-robotics [1]. The need of size reduction has led to forgo traditional engineering techniques for sensors fabrication in favor of clean room fabrication processes. The clean room facilities have enabled the production of the Micro Electro Mechanical Systems (MEMS). MEMS engineering can provide systems with much smaller details [2] than conventional techniques and can perform batch manufacturi ng, efficiently reducing costs and production time per unit.

MEMS force sensing can be divided into two main categories, namely elastic sensing and zero displacement sensing. The first one is the most widely reported in the literature with piezoresistive sensors [3, 4], fluidic sensors [5], capacitive sensors [6-8], MOSFET sensors [9], vision tracked sensors [10] and so on. Elastic sensors are

$\triangle$ Jonathan Cailliez

cailliez@isir.upmc.fr

Extended author information available on the last page of the article. based on a mechanical transformation of an external force into a displacement. The force measurement is deduced from the displacement measurement by the knowledge of mechanical suspensions stiffness. This method tends to provide smaller sensors with the need of little control electronic, leading to more integrated sensors. A trade-off between the measurement range and the resolution is often involved [11]. An increase of the sensor stiffness increases its measurement range at the cost of its resolution. In order to circumvent this drawback, a mechanical structure is used in [12] to change the sensor stiffness when the applied force exceeds a threshold value. One can also design an infinite stiffness sensor, called a zero displacement sensor.

The working principle of a zero displacement sensor, also referred here as an active sensor, is to hold the position of the probe at a fixed value despite of an external applied force. This is feasible thanks to a feedback control driving a set of actuators in order to compensate the applied external force. The force measurement is deduced from the actuator voltage or current. Active sensors have also the advantage of being able to provide quantitative force measurement without an accurate calibration of the suspensions. Some of zero displacement sensors have been reported in the literature. In 
[11] and [13], the sensor is composed of an electrothermal position sensor and an electrostatic comb drive actuator. However, the use of a traditional (i.e. not differential) comb drive configuration leads to a quadratic force/voltage relationship that involves control issues for the force measurement. In [14], the zero displacement force sensor includes a piezoresistive position sensor and an additional comb drive actuator, taking advantage of the equivalent 'negative stiffness' behavior of some electrostatic actuators, to adjust in real time the resonance frequency of the sensor. Overall, passive force sensors allow for more integrated sensors and can perform adequately provided their stiffness is higher than the maximum gradient of measured forces. They are also able to perform up to 6 DOF measurements [15], while active force sensors are more fit to measure forces with a high range of gradients.

The goal of this work is to present the modeling and experimental characterization of a high bandwidth MEMS force sensor with linear force-voltage actuation, folded flexure suspensions, while being as compact as possible A knowledge model of the sensor is proposed. Knowledge models are useful to study the influence of electromechanical parameters on the sensors performance and to have some feedback on conception.

A description of the MEMS structure is presented in Section 2. Section 3 deals with the electromechanical modeling of the sensor. The model describes the relationship between the sensor probe position and the actuation voltage of the comb drive actuator. The experimental protocol for the dynamic and the static characterization of the sensor is presented in Section 4. In Section 5 experimental data are analyzed and results are followed by several discussions.
The characterized model is used to perform some simulation as well as the synthesis on a controller for the sensor. A conclusion and future perspectives of the work finish the paper.

\section{Description of the MEMS structure}

The considered MEMS is presented in Fig. 1. A 2D plan and a picture of the MEMS with it's PCB are presented in Fig. 2.

The sensor is composed of a differential comb drive actuator, folded-flexure suspensions, a probe and six contact pads for the electrical connections as shown in Fig. 1. The goal was the design of a force sensor with a high linear range and a bandwidth superior to $2 \mathrm{kHz}$, to do so we made the choice of folded flexure suspensions. The choice of the actuator (number of comb drives and dimensions) was made so that it could be operated between $-70 \mathrm{~V}$ and $70 \mathrm{~V}$. The rest of the mechanical elements have been designed so that the mechanical resonant frequency fits our specifications. There are mainly 3 suspensions architectures used in MEMS devices: clamped clamped flexure, Crab-leg flexure and folded flexure design. If we consider the same flexure length and width and a force measurement in y direction, the clamped clamped suspensions have a high stiffness ratio $\mathrm{kx} / \mathrm{ky}$ equal to the square of the ratio length/width, but the linear range of ky is very low. The Crab-leg flexure design allows extending the linear range of ky but significantly reduces the ratio $\mathrm{kx} / \mathrm{ky}$. The folded flexure design, however, increases the linear range of ky compared to the clamped clamped design while it has the same ratio $\mathrm{kx} / \mathrm{ky}$ as for the clamped clamped flexures. Therefore, among these
Fig. 1 3D CAD view of the MEMS based force sensor. The folded-flexure suspensions are highlighted in yellow

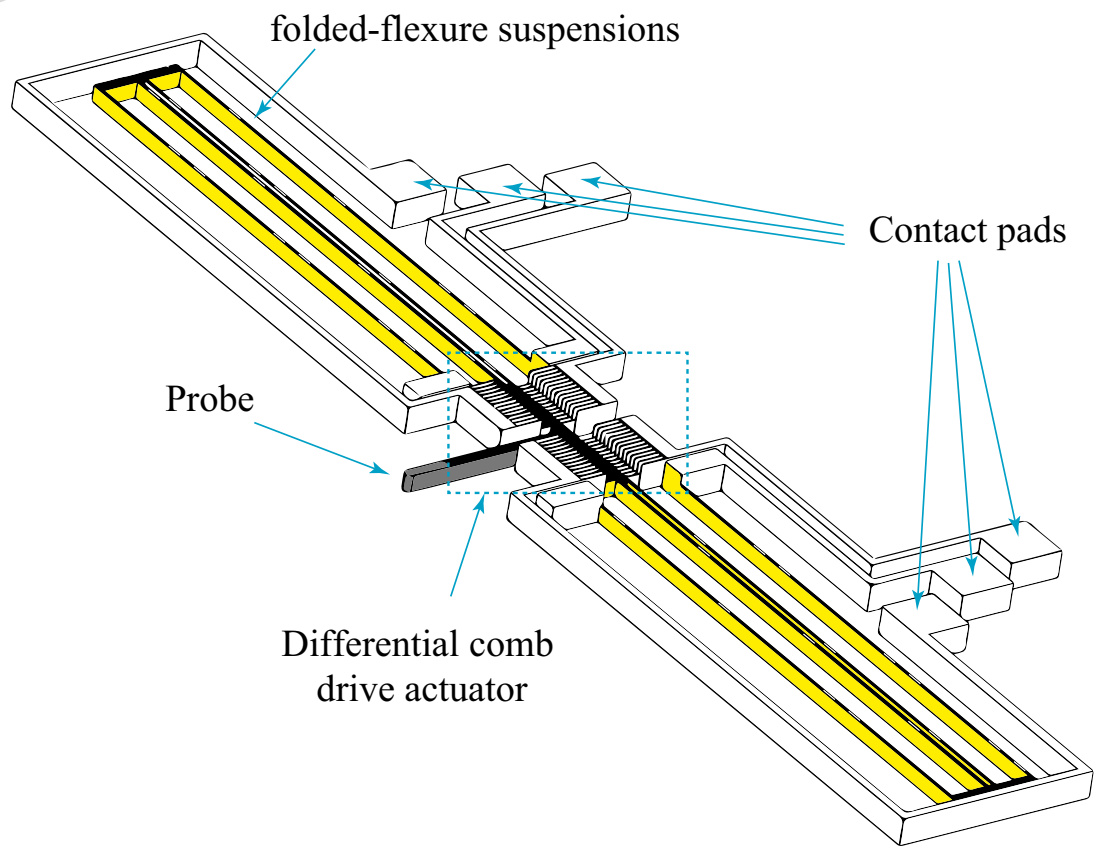


J Micro-Bio Robot

Fig. 2 MEMS based force sensor and enlarged view of the internal structure of the mechanical part. The movable structures are highlighted in red. Detail of one quarter of the comb drive actuator is presented above the global plan (the whole structure is $30 \mu \mathrm{m}$ thick)

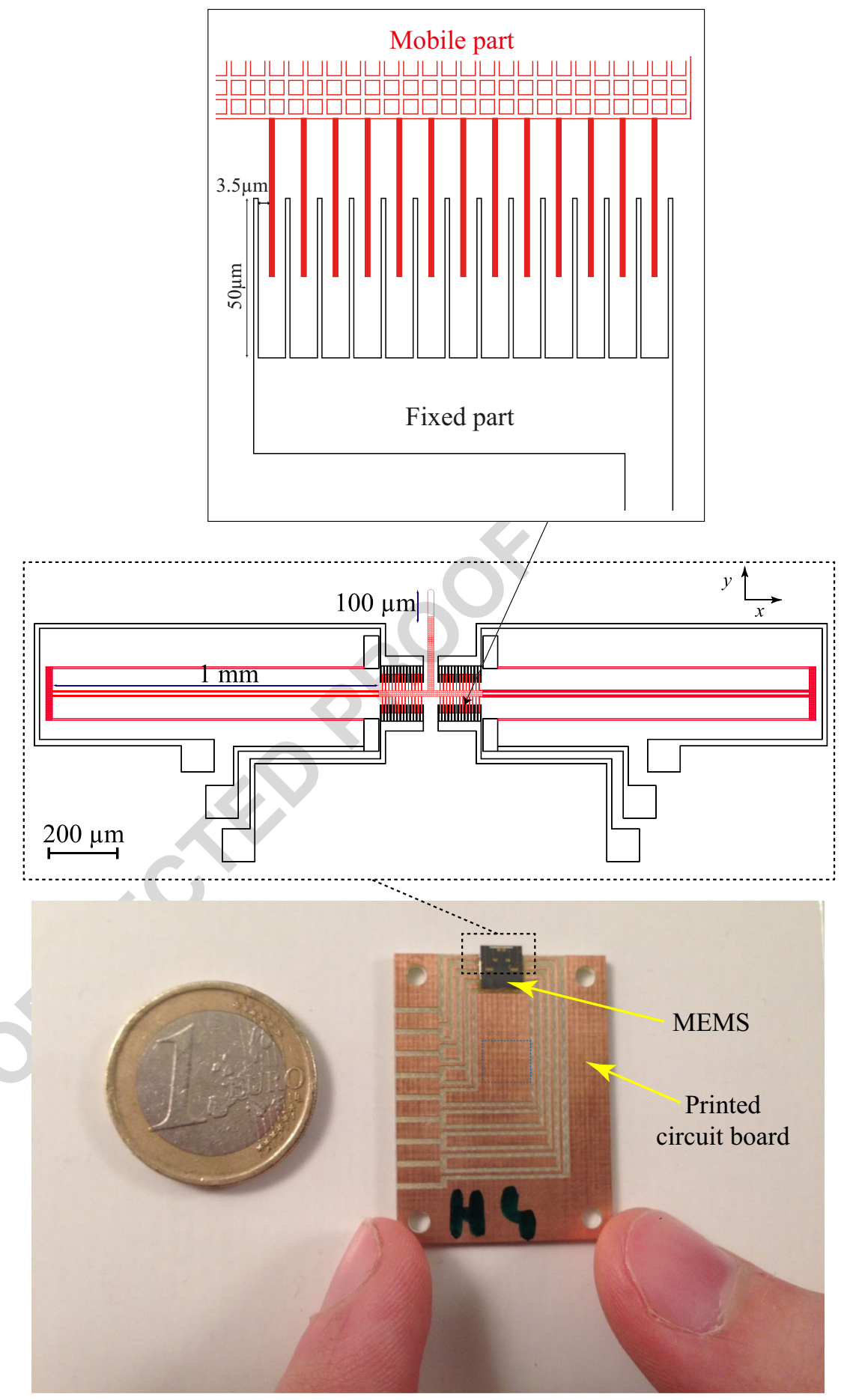

three design architectures, the folded flexure design is the best candidate when dealing with unidirectional force measurement in a wide linear operating range [16]. It has been monolithically fabricated on a silicon on insulator (SOI) wafer of $30 \mu \mathrm{m}$ thickness. Wire bonding has been used to connect the contact pads of the MEMS to a printed circuit board (Fig. 2).
The conception has been performed in our lab using a CAD software. However, as no cleanroom facilities are available in the lab, the MEMS were realized thanks to the help of the RENATECH platform and the IEMN lab (Institut d'éléctronique de microélectronique et de nanothechnologie). RENATECH is a french platform of nanofabrication. 
The nominal comb drive actuator includes 56 fixed fingers and 52 movable fingers, it can bee seen in Fig. 2. The gap spacing between the fingers is $g=3.5 \mu \mathrm{m}$. The suspensions have $1 \mathrm{~mm}$ length and $3.5 \mu \mathrm{m}$ width. The external part of the probe has a length of $100 \mu \mathrm{m}$ as shown in Fig. 2. The maximum actuation voltages of the differential comb drive actuator is 70 Volts. The linear displacement range of the probe is about $50 \mu \mathrm{m}$. The direction of motion of the probe is the $y$ direction.

\section{Dynamic modeling of the MEMS actuator}

For control purposes, a dynamic model of the sensor is needed. This section deals with the electromechanical modeling of the transfer between the probe displacement and the input voltages of the differential actuator.

\subsection{Electrical modeling of the differential electrostatic comb drives}

Let's consider an elementary finger pair of the comb drive actuator as depicted in Fig. 3. The movable fingers are represented by the electrode (2). The fixed fingers are represented by the electrodes (1) and (3). When no voltage is applied, the MEMS is designed to have $y_{1}=y_{2}=$ $y_{0}$, where $y_{1}$ and $y_{2}$ are the overlapping lengths between the electrodes (2) and (1) and the electrodes (2) and (3) respectively.

Hence, one can write:

$\left\{\begin{array}{l}y_{1}=y_{0}-y_{e} \\ y_{2}=y_{0}+y_{e}\end{array}\right.$

$y_{0}$ is the overlapping length when no voltage is applied and $y_{e}$ is the displacement of the movable finger in $y$ direction.

The electrostatic force exerted on the movable finger, in $y$ direction, in response to a voltage is equal to the gradient of the electrostatic energy stored by the system.

The stored energy can be expressed as follows :

$E=\frac{1}{2}\left(C_{12}\left(V_{1}-V_{2}\right)^{2}+C_{23}\left(V_{2}-V_{3}\right)^{2}+C_{13}\left(V_{1}-V_{3}\right)^{2}\right)$

$C_{i j}$ is the capacitance between the electrodes $(j)$ and $(i)$. $V_{i}$ is the voltage between an electrode $(i)$ and the electrical ground. Here, $C_{13}$ is considered equal to 0 (the electrical coupling between electrodes 1 and 3 is neglected).

By neglecting side effects, one can get:

$\left\{\begin{array}{l}C_{12}=2\left(y_{0}-y_{e}\right) \frac{N \epsilon_{0} \epsilon_{r} t}{g} \\ C_{23}=2\left(y_{0}+y_{e}\right) \frac{N \epsilon_{0} \epsilon_{r} t}{g}\end{array}\right.$

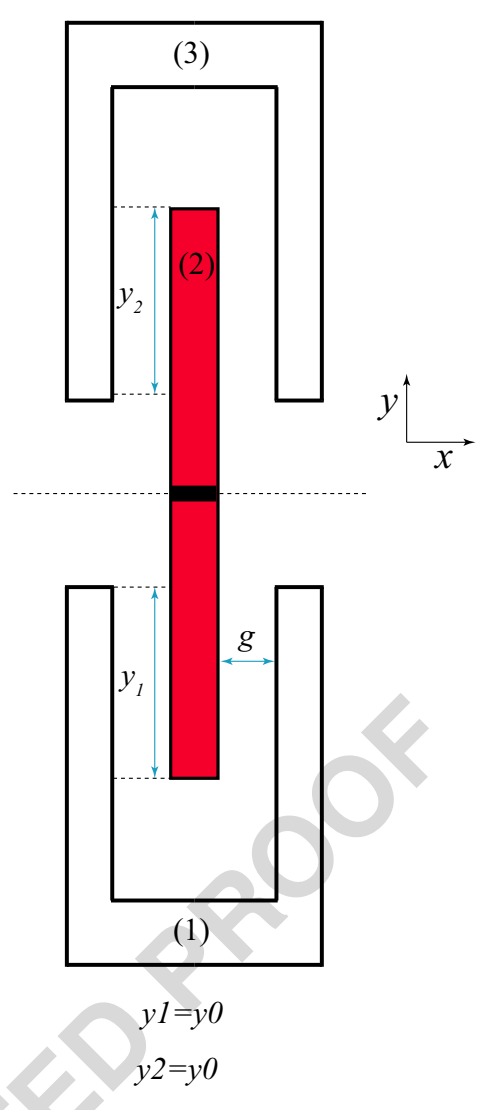

(a)

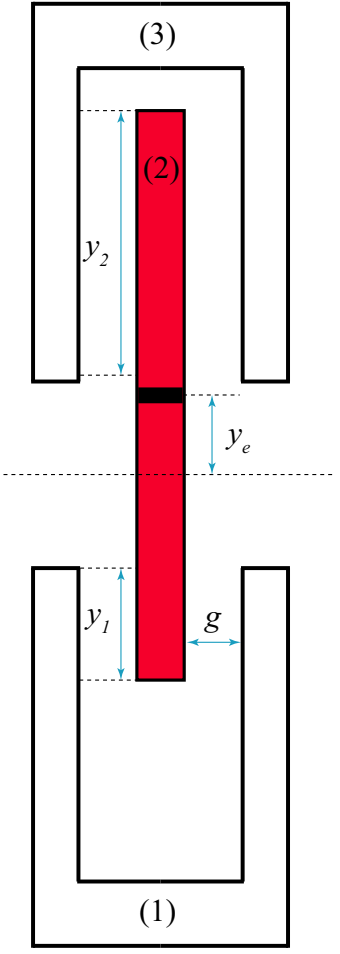

$y 1=y 0-y e$ $y 2=y 0+y e$

(b)
Fig. 3 Scheme of an elementary pair of fingers in the differential comb drive actuator when the movable fingers are at the initial position $y_{e}=$ 0 (a) and at a position $y_{e} \neq 0$. The movable fingers are represented by the electrode (2). The fixed fingers are represented by the electrodes (1) and (3)

$N=26$ is the total number of mobile fingers pairs, $\epsilon_{0}$ is the vacuum permittivity, $\epsilon_{r}$ the relative permittivity of air and $t=30 \mu \mathrm{m}$ the thickness of the electrodes.

Let us now consider the following gain:

$k_{c}=\frac{N \epsilon_{0} \epsilon_{r} t}{g}$

The electrostatic force can then be expressed as follows:

$$
\begin{aligned}
F & =-\frac{\partial E}{\partial y_{e}} \\
& =k_{c}\left(\left(V_{1}-V_{2}\right)^{2}-\left(V_{2}-V_{3}\right)^{2}\right)
\end{aligned}
$$

By setting $V_{1}=-V_{3}$, which is thereafter used to operate the force sensor, the Eq. 5 can be simplified as follows:

$F=4 k_{c} V_{3} V_{2}$

Using the numerical value of each parameter of the electrical model, and choosing $V_{3}=40 \mathrm{~V}$, the relationship between the electrostatic force $F$ that drives the sensor probe and the voltage $V_{2}$ is:
152 153 154 
J Micro-Bio Robot

Fig. 4 Schematic representation of the folded-flexure suspensions. The dashed rectangle shows the quarter model of the suspensions as shows in Fig. 5
$V_{1}$ will be set at $-40 \mathrm{~V}$ and $\mathrm{V} 3$ at $40 \mathrm{~V}$ for the experiments, $V_{2}$ becoming the only control signal. The linear force/voltage relationship which is one of the main advantages of the differential comb drive actuation is therefore demonstrated.

\subsection{Static mechanical modeling of the suspensions}

The aim of this section is to define a knowledge based model of the static force/deflection characteristic for the suspension structure. Let us recall that folded-flexure suspensions are designed for the MEMS sensor (Fig. 1). The bodies C1, C2, and C3 (Fig. 4) are supposed to be infinitely rigid and the flexible structures will be modeled with small displacement theory.

Due to the symmetry of the structure, the problem can be reduced by considering the quarter model of the suspensions as shown in Fig. 5. The dimensions of the beam 1 and that of the beam 2 are given in Table 1.

All the displacements are considered in $y$ direction. The displacement of the rigid body $\mathrm{C} 1$ will be supposed equal to the displacement of the point $\mathrm{A}$ of the beam 1 relatively to the point $\mathrm{B}\left(y_{a}\right)$ plus the displacement of the point $\mathrm{C}$ of the beam $2\left(y_{c}\right)$. The beam 1 will be supposed clamped at both ends and the beam 2 will be treated as simply clamped. The distance between the points $\mathrm{B}$ and $\mathrm{C}$ is equal to $18 \mu \mathrm{m}$.

Using Euler-Bernoulli beam theory, one is able to get the following Eqs. 8 and 9.

$$
y_{a}=\frac{F l_{1}^{3}}{48 E I}
$$

$y_{c}=\frac{F l_{2}^{3}}{12 E I}-\frac{F l_{1} l_{2}^{2}}{16 E I}$

With $\mathrm{E}$ the young modulus of silicon and I the area moment of inertia of the beam in the considered direction.

By combining Eqs. 8 and 9, the total displacement of the sensor probe is

$y_{p}=\frac{F}{12 E I}\left(\frac{l_{1}^{3}}{4}+l_{2}^{3}-\frac{3}{4} l_{1} l_{2}^{2}\right)$

The total stiffness $k$ of the suspension structure can then be deduced from the force/displacement relationship:

$k=\frac{y_{p}}{F}=\frac{12 E I}{\frac{l_{1}^{3}}{4}+l_{2}^{3}-\frac{3}{4} l_{1} l_{2}^{2}}$
Fig. 5 Quarter model of the suspensions (Dashed rectangle in Fig. 4)

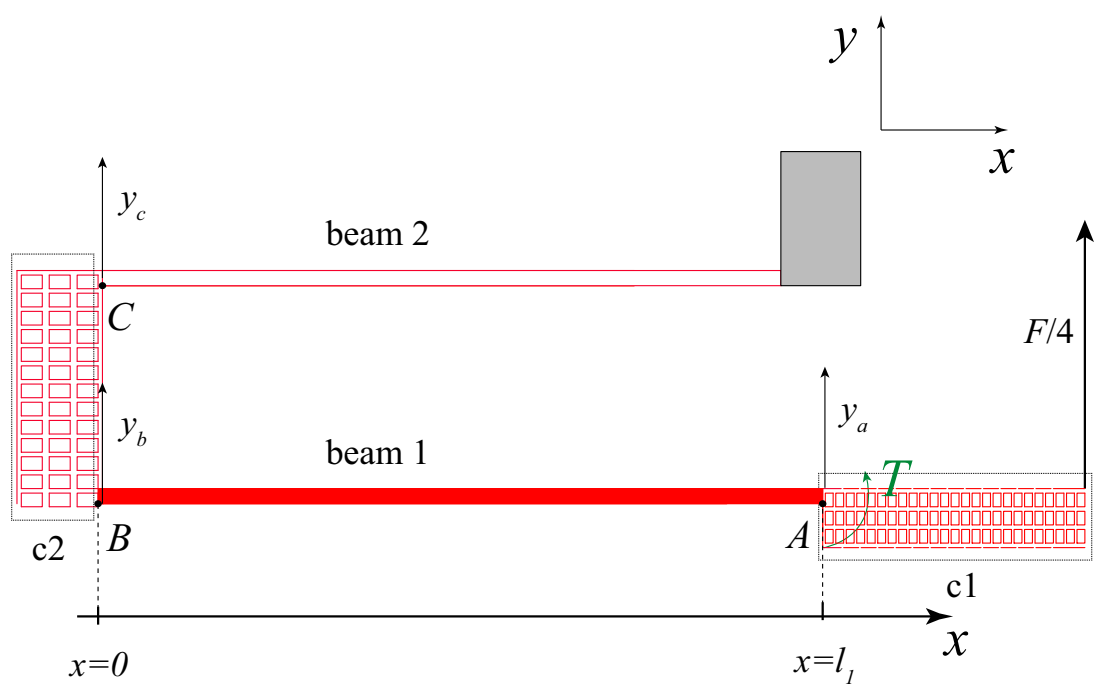


Table 1 Dimensions of the beam 1 and the beam 2 in the suspension structure

\begin{tabular}{llll}
\hline & width & thickness & length \\
\hline beam1 & $w=4.5 \mu m$ & $t=30 \mu m$ & $l_{1}=1 \mathrm{~mm}$ \\
beam2 & $w=4.5 \mu \mathrm{m}$ & $t=30 \mu \mathrm{m}$ & $l_{2}=0.965 \mathrm{~mm}$ \\
\hline
\end{tabular}

Taking into account the dimensions of the MEMS structure and the silicon Young modulus E = $127 \mathrm{GPa}$, the model (11) allows to compute $k=1.37 \mathrm{~N} / \mathrm{m}$.

The static force/displacement characteristic of the suspensions has been also analyzed using a computer-aided design (CAD) software. Several finite element analysis with different forces exerted on the probe in $y$ direction have been performed. The operating points have then been fitted to obtain the result of Fig. 6.

The finite element analysis leads to a stiffness $k=1.451$ $\mathrm{N} / \mathrm{m}$. The difference between this result and the one obtained by the knowledge-based model is equal to 5.58 $\%$, hence validating the hypothesis of the knowledge based model. However, both of these models consider perfect geometries, which is not necessary the case here due to the dimensions of the structure.

\subsection{Electro-mechanical dynamic model of the MEMS}

$U=V_{2}$ is the input of the system.

Using the Laplace transform of the Eq. 12, the transfer function $H(p)$ of the MEMS can be expressed as follows ( $p$ being the Laplace variable):

$H(p)=\frac{y_{p}}{U}=\frac{4 k_{c} V_{3}}{m p^{2}+\mu p+k}$

\section{Experimental characterization}

\subsection{Experimental setup}

As shown in Fig. 7, the experimental setup is composed of the MEMS sensor, voltage generators, a Digital Holographic Microscope (DHM) and a vibration isolation table. The DHM is used to measure the displacement of the mobile part of the MEMS in response to a voltage $U=V_{2}$. A beam of coherent light is emitted and focused on the MEMS. The intensity and phase of the reflected beam are recorded. The phase information is treated to get a 3D real time image of the observed structure.

\subsection{Dynamic characterization of the MEMS actuator}

Because of the high resonant frequency of the MEMS (relative to the DHM camera frequency), a stroboscopic unit has to be used to be able to track the position of the movable structure. For the experiment, a voltage of $40 \mathrm{~V}$ has been chosen for $V_{3}$, and the actuator is driven by a square wave $U=V_{2}$ at $50 \mathrm{~Hz}$. The stroboscope is set to get images at a frequency of $25 \mathrm{kHz}$. The first problem to solve is that the MEMS is not aligned with the microscope axes. The picture has to be rotated in order to align the movement direction with the image horizontal axis. To do so, the user selects 16 points in the image whose coordinates are known a priori. Then, 32 vectors are extracted from the 16 points.
Fig. 6 Static force/displacement finite element characteristic of the suspensions

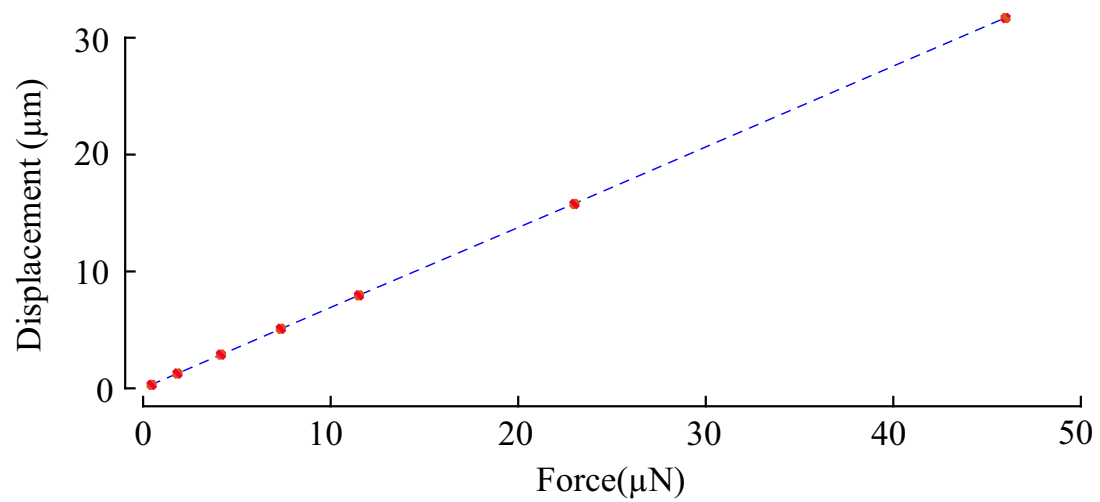


J Micro-Bio Robot

Fig. 7 Experimental setup for the characterization of the MEMS sensor. The stroboscopic unit is under the table
Let $A$ be the matrix containing the vector of coordinates in the image and $B$ the matrix containing the vector of the a priori coordinates such that $B=R \times A$ :

$A=\left[\begin{array}{ll}x_{a_{1}} & y_{a_{1}} \\ x_{a_{2}} & y_{a_{2}} \\ \vdots & \\ x_{a_{n}} & y_{a_{n}}\end{array}\right], B=\left[\begin{array}{ll}x_{b_{1}} & y_{b_{1}} \\ x_{b_{2}} & y_{b_{2}} \\ \vdots & \\ x_{b_{n}} & y_{b_{n}}\end{array}\right]$

The determination of $\mathrm{R}$ can be seen as an overdetermined system of equations. That means that no matrix $R$ is solution of this equation. However several solutions exist to provide a matrix $R$ that minimizes a cost function of $B-R A$. Here, the cost function will be quadratic. The chosen matrix $\mathrm{R}$ will be:

$R=B \times \operatorname{pinv}(A)$

Where pinv(.) is the Moore-Penrose pseudoinverse [17].

The rotation angle and scale ratio are extracted from $R$. The image is first rotated, missing pixels are recovered by a linear interpolation between neighboring points. The image is in grayscale, so pixels intensities range from 0 (black) to 255 (white). Pixels intensities are summed along the rotated image vertical axis. Figure 8 represents the sum of pixels intensities for one position of the MEMS probe. Similarly, Fig. 9 shows the same sum for several positions of the probe.

Let's consider the red curve in ROI represented by the dashed rectangle of Fig. 8 for a fixed position of the MEMS. The horizontal axis represents a pixel number in the horizontal direction. The vertical axis represents the sum of the intensities of the pixels that have the same horizontal position. This shape is characteristic of the probe. Around this shape, the intensity is constant, hence the corresponding offset can be removed, so the center of mass of the shape can be computed using Eq. 15 after removing the corresponding intensity offset. When the probe moves horizontally, the shape moves along with it by the same amount (with some distortion due to image blur).Therefore, the displacement of the probe can be estimated as the displacement of the center of mass of the ROI.

$y_{0}=\frac{\sum_{R O I} y \times\left(I(y)^{4}\right)}{\sum_{R O I}\left(I(y)^{4}\right)}$

$I(y)$ is the sum of the intensities of all the pixels that share the coordinate $y$ i.e. vertical sum of the intensities on $\mathrm{y}$, it is taken at the power four to increase the detection accuracy. $y$ can be expressed either as pixel number or converted to a position in microns using the scale ratio extracted from Eq. 14. Pattern tracking and data correlation are rendered impossible here due to high movement noise.

This method has been used to measure the step response of the MEMS probe experimentally (Fig. 10). These signals resulted in the identification of a second order model $H_{i}(p)$ describing the dynamic behavior of the MEMS. The step response of the model for a 4.5 Volts step input is also shown in Fig. 10.

The transfer function has been identified using MATLAB "system identification toolbox" on the system step response with $V_{3}=40 \mathrm{~V}$. It was performed looking for a transfer function model of order 2 with no zeroes The identified transfer function $H_{i}(p)$ is:

$H_{i}(p)=\frac{0.842 \times 10^{8}}{p^{2}+774.6 p+1.818 \times 10^{8}}$ 
Fig. 8 Top view of the MEMS with the DHM and example of curve showing vertical sum of pixels for a fixed position of the probe

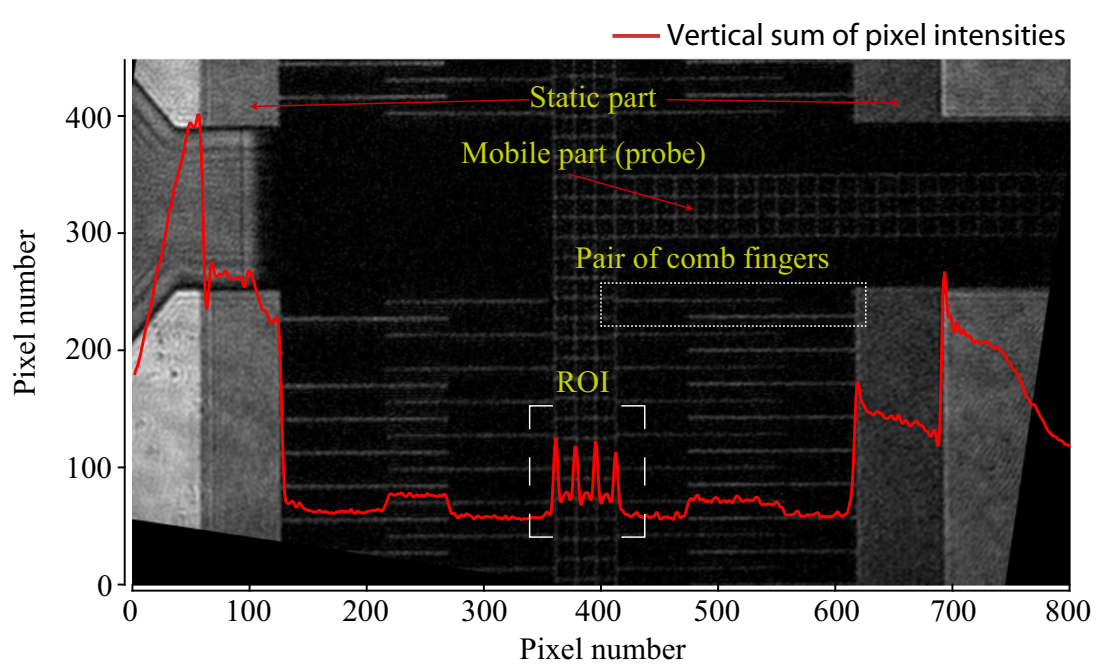

Its input and output are expressed in Volts and $\mu \mathrm{m}$ respectively. The resonant frequency of this model is 2.2 $\mathrm{kHz}$. This value is coherent with the one obtained through the finite element analysis of the MEMS. Bode plot of the identified transfer function is displayed in Fig. 11.

\subsection{Static characterization of the MEMS actuator}

The MEMS static characterization is essential to check the system linearity. To do so, the setup described in Section 4.2 is used. The system input is chosen to be sine wave at $5 \mathrm{~Hz}$. The position is recorded and plotted with respect to the input voltage. Result is shown in Fig. 12.

This curve can be modeled by first order polynomial. The experimental static gain of the MEMS is equal to $0.51 \mu \mathrm{m} / \mathrm{V}$. The static gain deduced from the fitted transfer function is $0.463 \mu \mathrm{m} / \mathrm{V}$. Figure 12 shows a characteristic that can be assimilated as hysteresis. However, as this nonlinearity is not described in the literature as being

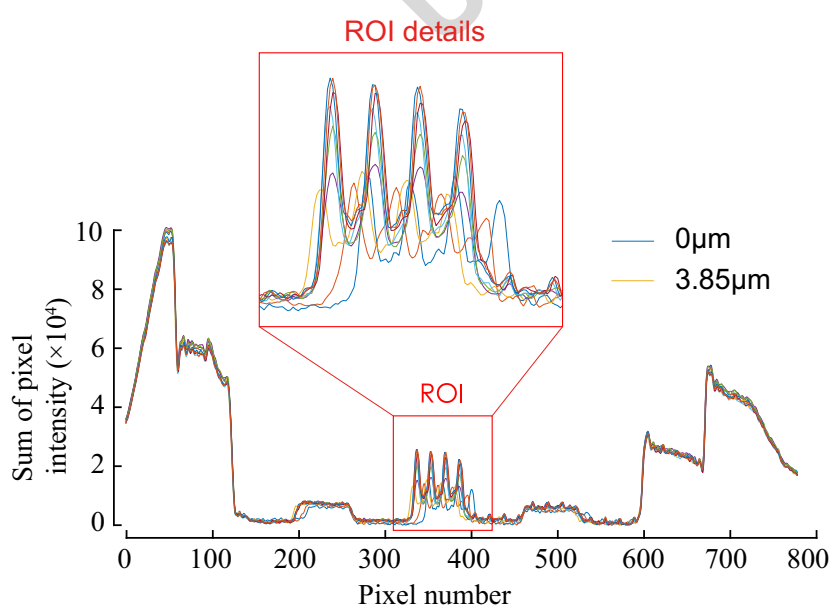

Fig. 9 Vertical sum of pixels for different values of displacements of the sensor probe (extreme values are shown with their legend) specific to electrostatic MEMS, we assume that this 316 phenomenon is not mainly due to a hysteresis and could 317 be due to some motion blur. Even though, this behavior is 318 not predominant and can be neglected in the modeling. The 319 non linearity error is lower than $0.4 \mu \mathrm{m}$ once measurement 320 artifacts have been removed, this error can be due to image 321 noise or outside vibrations. Experimental results differ by 322 a factor 2 from the ones presented in [18] because of a 323 faulty amplifier in the original setup which introduced an 324 amplification 2 times higher than expected.

\section{Simulation and test of the sensor in closed loop}

\subsection{Sensor simulation}

Now that the system transfer function has been characterized, a controller has to be designed for the sensor to operate in active mode. For the test in simulation to be possible, the coefficients of Eq. 13 have to be identified. However Eq. 16 only allows the identification of three of the four parameters. Because of this one of the four parameters has to be

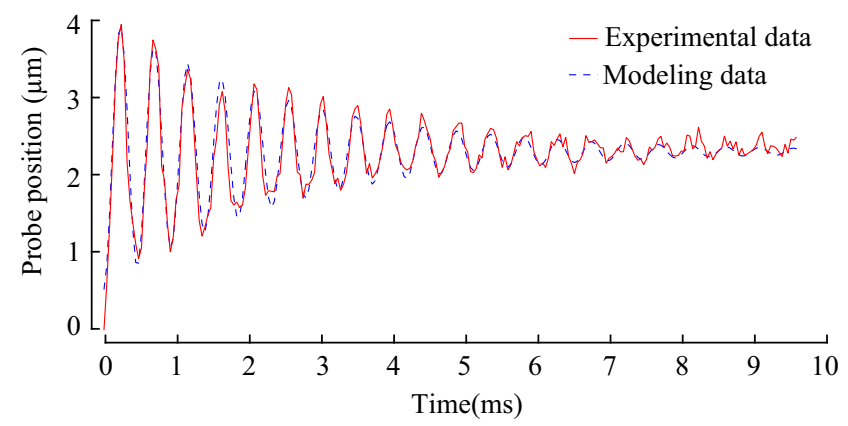

Fig. 10 Experimental and simulation step response of the MEMS sensor for a 4.5 Volts step input 

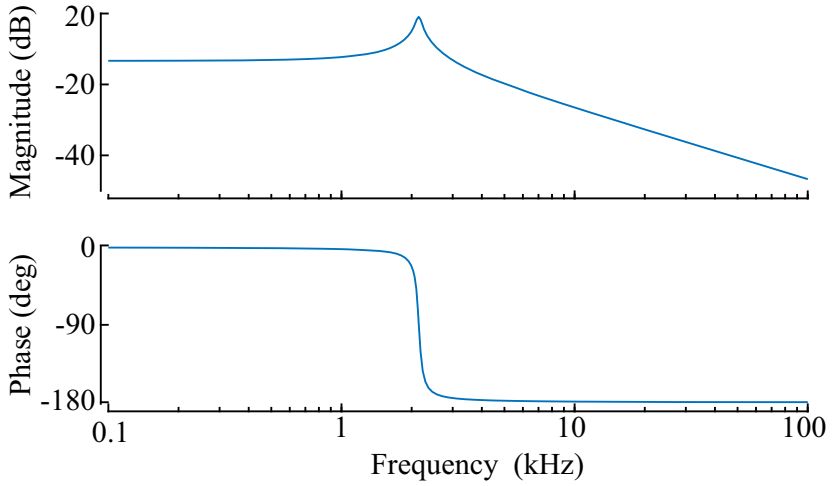

Fig. 11 Bode plot of the MEMS identified transfer function

identified by other means. There is little way to obtain $m$ and $v$ with enough accuracy, so either $k$ or $4 k_{c} V_{3}$ has to be taken from the knowledge model. Between both $4 k_{c} V_{3}$ is chosen.

$4 k_{c} V_{3}=0.316 \mu N / V$

This leads to :

$$
\begin{gathered}
m=3.7 \times 10^{-9} \mathrm{~kg}=3.7 \mu g \\
v=2.8 \times 10^{-6} \mathrm{~N} / \mathrm{m} \times \mathrm{s} \\
k=0.68 \mathrm{~N} / \mathrm{m}
\end{gathered}
$$

The difference between finite element analysis result and the identified $\mathrm{k}$ can be explained by several causes :

- The value of $k_{c} V_{3}$ is highly sensitive to the mechanical parameters of the comb drive, and the manufacturing processes can lead to some dimensions inaccuracies. Moreover the side effects (such as the capacitance between the finger front end and the part of fixed electrode in front of it) were neglected when computing the values of $C_{12}$ and $C_{23}$ and these hypothesis can not be entirely true [19].

- Finite element analysis at these scales is prone to errors as the geometries are supposed to be perfect and the materials are supposed to be homogeneous. However Deep Reactive Ion Etching often creates defects that are far from negligible at these scales.

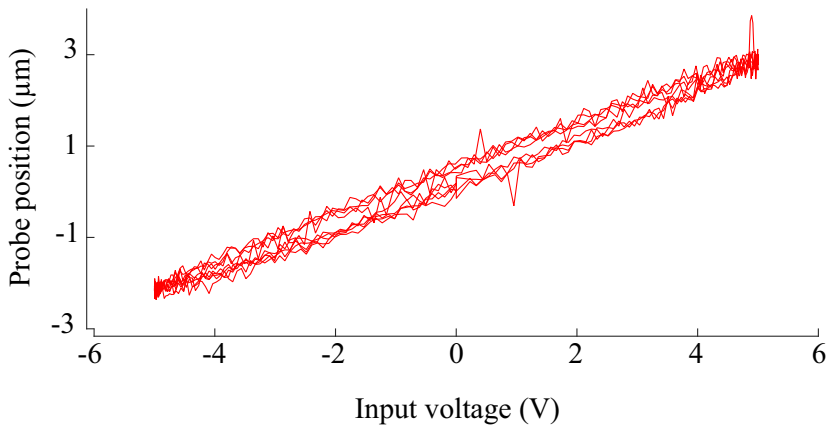

Fig. 12 Experimental static characteristic of the MEMS sensor

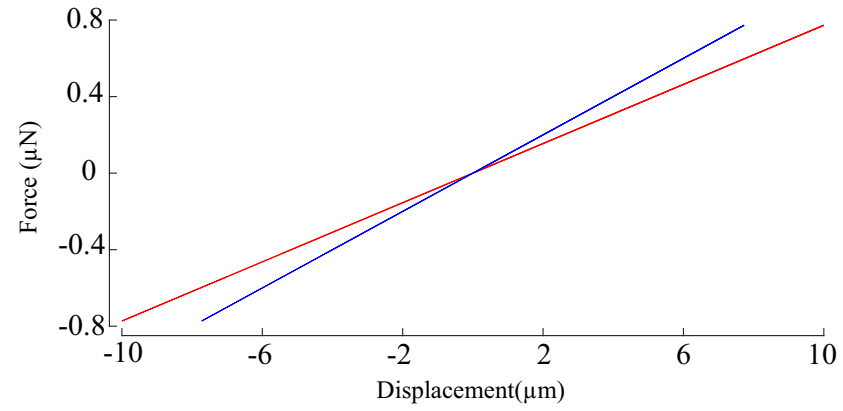

Fig. 13 Simulated force position characteristic of a $0.1 \mathrm{~N} / \mathrm{m}$ stiffness spring (blue) and estimated characteristic with the proposed sensor in passive mode (red)

\subsection{Differences between passive and active modes}

In passive mode, the sensor isn't actuated, any force applied on the sensor tip is translated into a displacement of the mobile part. In our case the displacement could be recorded thanks to a laser interferometer or by capacitive reading [20]. This mode only requires the identification of the flexures stiffness and is faster to implement than the active mode. However the sensor linearity depends on the flexure stiffness as well as the position measurement linearity. Furthermore, with this mode of operation, when trying to obtain force-position characteristics, the estimation is altered by the stiffness of the sensor flexures. In Fig. 13 are displayed the simulated force position characteristic of a spring with $0.1 \mathrm{~N} / \mathrm{m}$ stiffness acquired with the proposed sensor in passive mode only knowing the sensor position, as well as the actual characteristic.

One can notice a slight difference between the estimated and real force position characteristic. This is caused by the estimation of the characteristic of the spring in serial with the flexures equivalent spring. To compensate for this problem several solutions are possible :

- use a stiffer force sensor,

- subtract probe displacement from the sensor position,

- use the sensor in active mode.

Using the sensor in active mode can provide the advantage of having a virtually infinite stiffness and to measure far greater forces than would be allowed by the mobile part maximum displacement. First a controller has to be designed for the sensor, to do so, a PID controller has been implemented in simulation and fitted using Matlab PID tuner. During the design the constraints where a phase margin of $60^{\circ}$ and a bandwidth of $5 \times 10^{5} \mathrm{rad}^{-\mathrm{s}^{-1}}$ for the system in closed loop. The chosen bandwidth is of the same order of magnitude as the system resonant frequency as increasing it further would not improve the sensing performance. Figure 14, shows the normalized step response of the sensor with and without the controller. Position of 


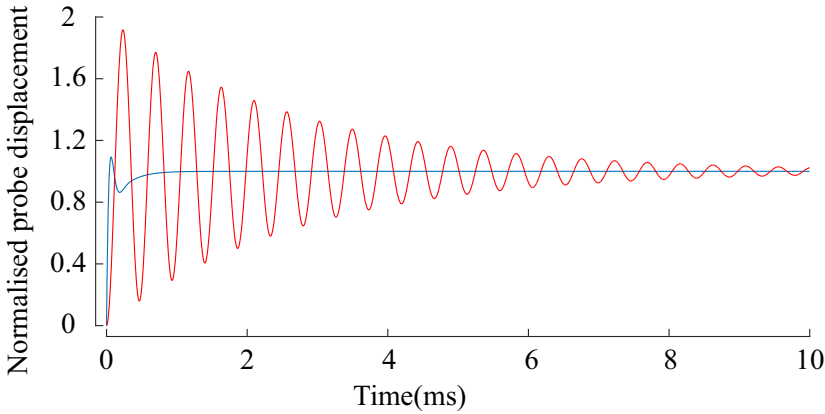

Fig. 14 Simulated normalized position of the sensor's probe in open loop (red) and in closed loop (blue)

the sensor's probe and PID output when a force of $10 \mu \mathrm{N}$ is applied to the sensor are displayed in Fig. 15. On this curve one can notice a slight transient behavior of the sensor. However, this transient displacement is inferior to the sensor maximum displacement $(20 \mu \mathrm{m})$ thus allowing for the estimation of forces superior to the ones measurable in passive mode. furthermore, once steady state is reached the sensor mobile part is kept at its initial position.

\subsection{Experimental test of the sensor}

The actual experimental setup lacks the ability to measure the displacement of the sensor's probe in real time. Consequently, to validate the simulation results and the controller that has been synthesized, a simulation is run with the model of the sensor and the controller output is recorded. This record is played in a loop in order to be able to perform a stroboscopic analysis similar to the one performed in Section 4.2. However, the actual experimental setup lacks the capability to play the data at a sufficient speed. To be able to test the validity of the proposed MEMS model, a discrete time PID has been designed for the sensor with a $10 \mathrm{kHz}$ sampling frequency, with design requirements of a $2 \mathrm{~ms}$ closed loop response and a $60^{\circ}$ phase margin. The closed loop response time

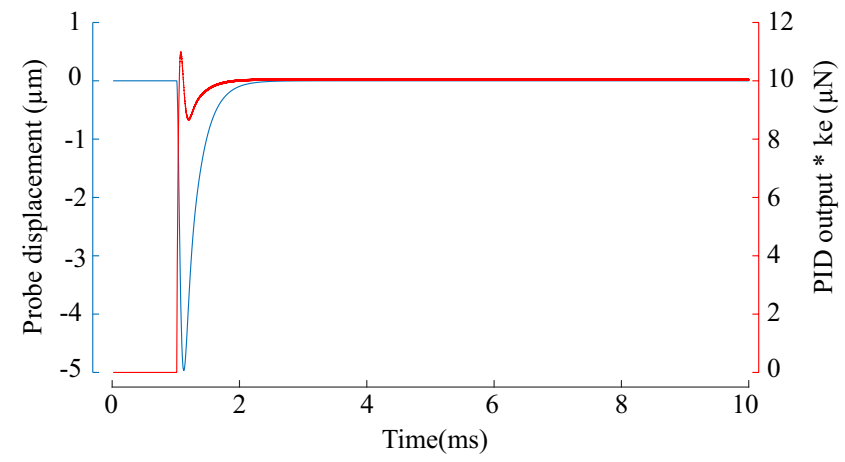

Fig. 15 Simulated probe displacement (blue) and active sensor reading (red) for an applied force of $10 \mu \mathrm{N}$

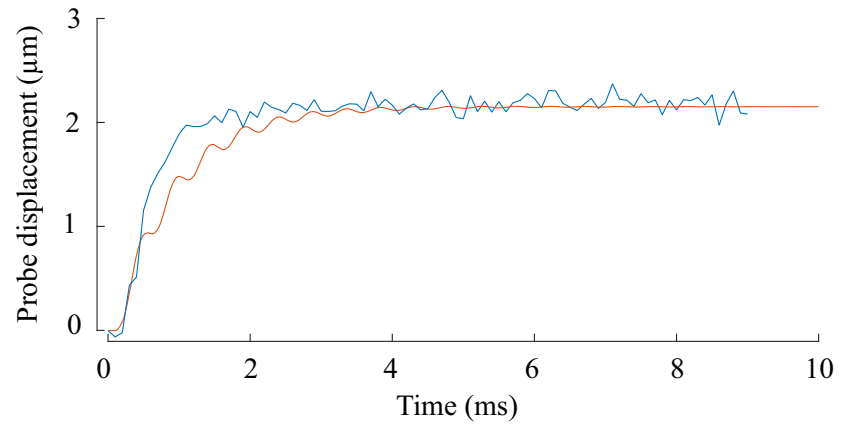

Fig. 16 Experimental probe displacement (blue) and simulation result (orange) for a step reference of $2 \mu \mathrm{m}$

is a compromise between system speed and the likeness of having undetectable oscillations between samples. The resulting sensor trajectory is displayed in Fig. 16, alongside with the predicted results. The results show the sensor's ability to reach a position of $2 \mu \mathrm{m}$, which corresponds, based on the value of $\mathrm{k}$ deduced from the transfer function, to a force of $1.36 \mu N$.

The experimental results fit the simulation results reasonably well, the discrepancies can be explained by the measurement noise due to the image motion blur.

\section{Conclusion}

Based on a differential comb drive actuator and a foldedflexure suspension, a new MEMS force sensor is proposed in this paper. By design, its suspension is arranged to constrain the sensor probe within a single direction. Whilst, the differential actuator is chosen to provide a linear force/voltage relationship where force and probe position are independent. Furthermore, because of the suspension linearity, the sensor can be used as a force-sensing positioner with $\pm 20 \mu \mathrm{m}$ displacement range, while retaining its mechanical performance independently from the operating point. In view of using a zero displacement principle (balance principle), a control oriented electromechanical model is driven. Preliminary experimental characterization validates this model in static and dynamic operating modes using a stroboscopic measurement system. The sensor shows a promising potential with a high bandwidth and a great linear force measurement range, thus allowing for future work on the measurement of forces with great dynamics. Furthermore, because of the zero displacement sensing, this sensor is usable for the measure of forces with high gradients without compromises on its resolution or accuracy. The simulations results have shown the possibility to control the sensor in active mode.

Future work will focus on the sensor control in real time by the use of a laser interferometer and the sensor 
calibration, as well as the controller implementation with analog components. This sensor will be used for instance to measure the stiffness of muscular cells in order to differentiate cancerous cells from healthy ones.

Acknowledgment This work has been partially sponsored by the French National Research Agency project MultiFlag (Grant No. ANR16-CE33-0019) and COLAMIR (Grant No. ANR-16-CE10-0009) and the project Robotex Equipment of Excellence (ANR-10-EQPX-44). The MEMS were realized thanks to the help of the RENATECH platform and the IEMN lab (Cité Scientifique - Avenue Poincaré BP 60069, 59652 Villeneuve d'Ascq Cedex).

\section{References}

1. Chaillet N, Régnier S (2013) Microrobotics for micromanipulation, isbn: 9781848211865

2. Koch SJ, Thayer GE, Corwin AD, de Boer MP (2006) Micromachined piconewton force sensor for biophysics investigations. Appl Phys Lett 89(17):173901

3. Kohyama S, Takahashi H, Yoshida S, Onoe H, Shoji KH, Tsukagoshi T, Takahata T, Shimoyama I (2017) Mems force and displacement sensor for measuring spring constant of hydrogel microparticles. In: International conference on micro electro mechanical systems (MEMS), pp 1040-1043

4. Mei T, Li WJ, Ge Y, Chen Y, Ni L, Chan MH (2000) An integrated mems three-dimensional tactile sensor with large force range. Sensors Actuators A Phys 80(2):155-162

5. Gutierrez CA, McCarty C, Kim B, Pahwa M, Meng E (2010) An implantable all-parylene liquid-impedance based mems force sensor. In: 2010 IEEE 23rd international conference on micro electro mechanical systems (MEMS), pp 600-603

6. Boudaoud M, Le Gorrec Y, Haddab Y, Lutz P (2015) Gain scheduling control of a nonlinear electrostatic microgripper: design by an eigenstructure assignment with an observer-based structure. IEEE Trans Control Syst Technol 23(4):1255-1267

7. Boudaoud M, Haddab Y, Le Gorrec Y (2013) Modeling and optimal force control of a nonlinear electrostatic microgripper. IEEE/ASME Trans Mechatronics 18(3):1130-1139

8. Boudaoud M, De Faria MG, Gorrec YL, Haddab Y, Lutz P (2014) An output feedback lpv control strategy of a nonlinear electrostatic microgripper through a singular implicit modeling. Control Eng Pract 28:97-111
9. Gao W, Zhao L, Jiang Z, Xia Y, Guo X, Zhao Z, Zhao Y, Sun D (2017) A novel mems force sensor based on laterally movable gate array field effect transistor(lmgafet). In: International conference on nano/micro engineered and molecular systems (NEMS), pp 723-727

10. Guelpa V, Prax J, Vitry Y, Lehmann O, Dehaeck S, Sandoz P, Clévy C, Le Fort-Piat N, Lambert P, Laurent GJ (July 2017) 3d-printed vision-based micro-force sensor dedicated to in situ sem measurements. In: 2017 IEEE international conference on advanced intelligent mechatronics (AIM), pp 424-429

11. Coskun MB, Moore S, Moheimani SR, Neild A, Alan T (2014) Zero displacement microelectromechanical force sensor using feedback control. Appl Phys Lett 104(15):153502

12. Chen W, Jiang J, Liu J, Chen W (July 2013) A mems based sensor for large scale force measurement. In: 2013 IEEE/ASME international conference on advanced intelligent mechatronics, pp 1278-1283

13. Moore SI, Coskun MB, Alan T, Neild A, Moheimani SOR (2015) Feedback-controlled mems force sensor for characterization of microcantilevers. J Microelectromech Syst 24(4):1092-1101

14. Maroufi M, Alemansour H, Moheimani SOR (2017) A closedloop mems force sensor with adjustable stiffness. In: IEEE conference on control technology and applications (CCTA)

15. Beyeler F, Muntwyler S, Nelson BJ (2009) Design and calibration of a microfabricated 6-axis force-torque sensor for microrobotic applications. In: 2009 IEEE international conference on robotics and automation, pp 520-525

16. Legtenberg R, Groeneveld AW, Elwenspoek M (1996) Combdrive actuators for large displacements. J Micromech Microeng 6(3):320

17. Courrieu P (2008) Fast computation of moore-penrose inverse matrices. CoRR arXiv:0804.4809

18. Cailliez J, Boudaoud M, Mohand-Ousaid A, Weill-Duflos A, Haliyo S, Régnier S (2018) Modeling and experimental characterization of an active mems based force sensor. In: International conference on manipulation, automation and robotics at small scales (MARSS), pp 91 (6), Nagoya, Japan, p 2018

19. Jaecklin VP, Linder C, de Rooij NF, Moret JM (1992) Micromechanical comb actuators with low driving voltage. J Micromech Microeng 2(4):250

20. Sun Y, Fry SN, Potasek DP, Bell DJ, Nelson BJ (2005) Characterizing fruit fly flight behavior using a microforce sensor with a new comb-drive configuration. J Microelectromech Syst 14(1):4-11

Publisher's Note Springer Nature remains neutral with regard to jurisdictional claims in published maps and institutional affiliations.

\section{Affiliations}

Jonathan Cailliez $^{1} \cdot$ Mokrane Boudaoud $^{1} \cdot$ Abdenbi Mohand-Ousaid $^{2} \cdot$ Antoine Weill-Duflos $^{3} \cdot$ Sinan Haliyo $^{1}$. Stéphane Régnier ${ }^{1}$

Mokrane Boudaoud mokrane.boudaoud@sorbonne-universite.fr

Abdenbi Mohand-Ousaid abdenbi.mohand@femto-st.fr

Antoine Weill-Duflos antoine.weill-duflos@mcgill.ca

Sinan Haliyo

sinan.haliyo@ sorbonne-universite.fr
Stéphane Régnier

stephane.regnier@upmc.fr

1 Sorbonne Université, UMR 7222, ISIR, F-75005 Paris, France

2 FEMTO-ST Institute, AS2M Department, University Bourgogne Franche-Comte / UFC / CNRS UMR-6174/ENSMM, 24, rue Alain Savary, 25000 Besançon, France

3 McGill University, Centre for Intelligent Machines, 3480 Rue University, Montréal, Québec H3A 0E9, Canada 